\title{
Sapphyrin-Nanotube Assemblies
}

\author{
Peter J. Boul $\uparrow, \uparrow$, Dong-Gyu Cho $\dagger$, G. M. Aminur Rahman $\ddagger$, Manuel Marquez $§$, Zhongping \\ Ou\|, Karl Kadish $\|$, Dirk M. Guldi ${ }^{\ddagger}$, and Jonathan, L. Sessler ${ }^{\dagger}$ \\ University of Texas at Austin, Department of Chemistry and Biochemistry, 1 University Station \\ A5300, Austin, TX. 78712, INEST Group Postgraduate Program, Philip Morris USA, Richmond, VA \\ 23234, the Institute for Physical and Theoretical Chemistry, Friedrich-Alexander-Universität \\ Erlangen-Nürnberg, 91058 Erlangen, Germany, Research Center, Philip Morris USA, 4201 \\ Commerce Road, Richmond, VA 23234, and Department of Chemistry, University of Houston, \\ Houston, TX 77204-5003
}

\begin{abstract}
Single wall carbon nanotubes (SWNTs) bind strongly to sapphyrins, quintessential pentapyrrolic "expanded porphyrin" macrocycles, through $\pi$-stacking interactions. The specific use of a functionalized sapphyrin diol yields stable water-suspendable nanotubes and also permits the formation of well-defined assemblies in ionic liquids. The absorption and steady-state fluorescence spectra of the resulting non-covalently functionalized nanotube complexes have been analyzed in aqueous and ionic liquids, yielding a description of the photophysical properties of the nanotubesapphyrin complexes as donor-acceptor species for light-harvesting.
\end{abstract}

\section{Introduction}

The directed assembly of functional components to single wall carbon nanotube surfaces in application to medicinal, ${ }^{1}$ electronic, ${ }^{2}$ and light harvesting materials ${ }^{3}$ remains a challenge in the design of nanotube superstructures. Of particular interest in this regard is the non-covalent functionalization of carbon nanotube materials. Such modifications allow for the development of nanometric constructs without irreversibly changing or damaging the electronic structures specific to these conducting and semiconducting quantum wires. The ability to control the assembly of small organic molecules on nanotube surfaces without recourse to extensive sonication is further advantageous in that the molecular integrity of the materials can be spared from sonication damage.

The photophysical properties of the nanohybrids formed between porphyrins and single wall nanotubes (SWNTs) have been characterized through steady-state and time-resolved spectroscopic methods, measurements that have provided support for porphyrin-nanotube donor-acceptor interactions. ${ }^{4}$ In contrast, expanded porphyrins display different chemical and physical properties relative to their tetrapyrrolic cousins. Sapphyrins, well-characterized pentapyrrolic macrocycles, ${ }^{5}$ are particularly attractive candidates for complexation to SWNTs as they present larger $\pi$ surfaces than do typical tetrapyrrolic macrocycles. As compared to porphyrins, sapphyrins are fluorescent 6 through excitation at lower energy making them particularly attractive as mimics of naturally occurring chlorophylls, the key electron donors

șessler@mail.utexas.edu.

The University of Texas at Austin

$\hat{\jmath}_{\text {INEST Group Postgraduate Program }}$

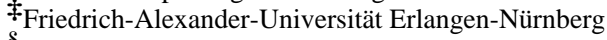

$\S_{\text {Research Center, Philip Morris USA }}$

"The University of Houston 
in photosynthesis. ${ }^{7}$ In this interest, non-covalent systems of sapphyrin have been designed based on both oligonucleotides and carboxylate anion recognition as models for the processes occurring in natural photosynthetic systems. ${ }^{8}$ Here, we demonstrate the use of a readily available sapphyrin diol $\mathbf{1}^{9}$ (cf. Scheme 1) for the generation of non-covalent light harvesting donor-acceptor ensembles with SWNTs in water, and in the ionic liquid, 1-butyl-3methylimidazolium hexafluorophosphate (BMIM-PF6), reported to be among the most effective ionic liquids for exfoliation of SWNTs ${ }^{10}$ and as solvents for covalent functionalization of SWNTs. ${ }^{11}$

To the extent that sapphyrin might be used to generate noncovalent adducts with SWNTs, it provides a set of supramolecular constructs with built-in antennae for light capture and thus the creation of biomimetic nanotube-based photovoltaic devices. In this report we detail the first steps towards the production of such devices, namely the characterization of an initial set of sapphyrin-functionalized nanotube complexes. These complexes demonstrate rapid decay of sapphyrin excited states, something that does not occur for sapphyrin solutions in the absence of nanotubes. The presence of the sapphyrin radical anion peak at $840 \mathrm{~nm}$ in the transients indicated an electron transfer from the nanotubes to the sapphyrin. As detailed below, these new complexes were characterized through spectroscopic and microscopic means.

\section{Experimental Methods}

\section{Chemical Compounds}

The sapphyrin described in this paper was synthesized as published previously. ${ }^{10} 1$-Butyl-3methylimidazolium hexafluorophosphate was purchased from Sigma-Aldrich Chemical Co. Highly Oriented Pyrolytic Graphite used in atomic force microscopy was purchased from Structure Probe Inc. SWNTs, produced by the HiPco process, were purchased from Carbon Nanotechonologies Inc.

\section{Preparation of Nanotube Complexes}

As illustrated in Scheme 1, supramolecular functionalization of SWNTs by sapphyrin was effected in both aqueous media and BMIM-PF6. In water, functionalization was typically achieved through a three step procedure. In the first step about $0.4 \mathrm{mg}$ of sapphyrin diol and $0.4 \mathrm{mg}$ of purified HiPco SWNTs were sonicated in a Bransonic 52 (112 Watt) sonicating bath for 90 minutes. The resulting suspension was centrifuged at $12,000 \mathrm{rpm}$ in a bench top Eppendorf Minispin centrifuge. The resulting solid was then resuspended with nanopure water through 45 minutes of sonication. The original supernatant was also saved and analyzed using atomic force microscopy (AFM), as discussed below.

In contrast to the method described above, supramolecular nanotube-sapphyrin complexes could be obtained in the ionic liquid, BMIM-PF6, without recourse to sonication at any point in the procedure. To create donor-acceptor complexes in this solvent, $0.4 \mathrm{mg}$ of sapphyrin diol, $0.4 \mathrm{mg}$ of HiPco SWNTs, and $1.5 \mathrm{ml}$ of the ionic liquid in question were ground with a mortar and pestle for 10 minutes. This part of the procedure served to exfoliate the nanotubes, thus permitting the sapphyrin to come in contact with the newly exposed annotate surfaces. The rest of the preparation follows that described for the sapphyrin suspensions in water, with the exception that the sonication step is replaced by stirring.

\section{Analysis of nanotube-sapphyrin complexes}

In order to get a qualitative indication of the relative amount of individual nanotubes and nanotube bundles which were present in the aqueous suspensions, AFM measurements were carried out for both the resuspended pellet and the supernatant. The nanotube suspensions, produced according to the procedure outlined above, were spin-coated onto Highly Oriented 
Pyrolytic Graphite (HOPG). After spin-coating, the wafers were washed with electronics grade 2-propanol and air dried. The analyses were carried out in tapping mode using a Digital Instruments Dimension 3100 scanning probe microscope with a Nanoscope IV controller. Two samples were analyzed. The first sample consisted of nanotubes suspended from the pellet after the centrifugation step. The second sample contained nanotubes obtained from the supernatant following the centrifugation. In both cases, a combination of SWNT bundles and individual nanotubes was observed (see Figure 1).

Three spectroscopic techniques were used to probe the nature of the interaction between sapphyrin and the nanotubes: (1) UV/VIS/NearIR spectroscopy, (2) fluorescence spectroscopy, and (3) time-resolved femtosecond spectroscopy. All of these methods support one another in that they provide evidence of electronic and face-to-face stacking interactions between the sapphyrin and the nanotubes, as well as rapid excited state deactivation, via a process wherein the sapphyrin chromophore acts as an excited state electron acceptor and the nanotube as the electron donor. Sapphyrin, 1, was also analyzed by cyclic voltametry and pulsed radiolysis to obtain baseline data for comparison with that arising from the transient absorption analyses.

\section{Results and Discussion}

Absorption spectroscopy is a particularly useful method of characterizing sapphyrincontaining species. This is because the observation of a significant red shift in the Soret-type band would provide prima facie evidence for a strong electronic interaction between the sapphyrin $\pi$ surface and the nanotubes. Solutions of sapphyrin diol 1 in water at a $13.5 \mu \mathrm{M}$ concentration are characterized by three different Soret-like absorption bands. These have been assigned previously and are considered to be due to the monomer $(450 \mathrm{~nm})$, the dimer (425 $\mathrm{nm}$ ), and higher aggregates $(390 \mathrm{~nm}) .{ }^{12}$ While these peaks are observable in the spectrum of solutions of the free sapphyrin 1, the red shift in the sapphyrin Soret-type band of sapphyrin when complexed to nanotubes complexed is present regardless of the concentration of the nanotube material employed, at least within readily testable limits (Figure 2).

On the basis of this latter observation, we propose that the nanotube acts as a template, facilitating the supramolecular attachment of the monomeric sapphyrin diol 1 on the surface of the nanotube. Consistent with this hypothesis is the fact that there are no peaks corresponding to the dimer or aggregate forms of sapphyrin in the spectra of samples wherein the sapphyrin diol is monomerized due to the addition of a surfactant or via presumptive binding to the single wall nanotubes. Moreover, a linear dependence of the optical density was observed with dilutions of the sapphyrin nanotube mixtures down to the point where the concentration of the nanotubes fell below what could be detected reliably. Such observations reduce concerns that the sapphyrin complexed nanotubes precipitate upon increasing dilution or are stabilized by nonspecific interactions involving free sapphyrin over the timeline of the experiments (on the order of several hours).

It can be observed in Figure 3, that the van Hove singularities of the nanotubes are largely present and co-exist with features specific to sapphyrin. In ionic liquids there is some free sapphyrin that remains unbound to the nanotube, just as there is sapphyrin involved in $\pi$ stacking. The resulting spectra, therefore, are characterized by the presence of more than one Soret-type band (including ones ascribable to various sapphyrinnanotube aggregates). However, as will be described later, the time dependent nature of these bands allows them to be resolved into nanotube-bound and nanotube-unbound sapphyrin species.

Fluorescence emission spectra of the nanotubesapphyrin supramolecular complexes and of the starting sapphyrin diol were taken separately (Figure 4). These spectra are consistent with the proposal that the sapphyrin is indeed in supramolecular contact with the nanotube. In particular, while the background of the nanotube sample was significantly higher than that of sapphyrin 
due to SWNT fluorescence (cf. Figure 3), the intensity of the fluorescence emission peak is diminished by about a factor of seven. Additionally, the maximum of this fluorescence signal is blue shifted. Finally, excitation of sapphyrin-nanotube complexes at $477 \mathrm{~nm}$ also reveals that the intensity of the shifted Soret signal is reduced. Thus, the combined changes in the absorption and fluorescence emission spectroscopic features are consistent with the hypothesis that the sapphyrin vibrational modes undergo a change in energetics when this macrocycle comes into supramolecular contact with single wall nanotubes. Specifically, it is suggested that binding to the SWNT surface leads to an overall change in the wavelength of the Soret-type band as the result of changes in the free energy of binding.

In order to compare free sapphyrin with nanotube bound sapphyrin, femtosecond transient spectroscopic studies were carried out. Both sapphyrin species were analyzed separately because predicative data for sapphyrin alone had not previously been recorded under conditions analogous to those used to prepare and study the sapphyrin-nanotube complexes. Considering the latter control experiments first, it was found that following $387 \mathrm{~nm}$ flash photolysis of free sapphyrin 1 in water in the absence of SWNTs new transients develop at $467 \mathrm{~nm}$ and $494 \mathrm{~nm}$ that are readily assigned to excited states of sapphyrin. The corresponding bleaching features at $449 \mathrm{~nm}$ - a consequence of reducing the population of the ground state - were found to exhibit little appreciable decay over the time scale of our experiments ( $1500 \mathrm{ps})$. This was true for measurements made in water, as well as those carried out in BMIM-PF6. The Q-bands of the sapphyrin were also found to bleach following femtosecond photolysis; unfortunately, however, these features were found to be of little use in terms of making comparisons to nanotube complexes (vide infra) due to the influence of the van Hoves on the position and kinetics of the Q-bands.

When a suspension of the nanotube-sapphyrin complex is subject to the same photoexcitation process as discussed above for sapphyrin without nanotubes, an analogous transition is found that again corresponds to Soret band bleaching, albeit red-shifted to $480 \mathrm{~nm}$ in water. So as to measure the kinetics without the interference of bleaching at $480 \mathrm{~nm}$, the excited state decays were analyzed at $500 \mathrm{~nm}$. A first order exponential decay, with a lifetime of $9.4 \pm 1.4 \mathrm{ps}$, was observed for nanotube-sapphyrin complexes in water, with the corresponding value being 27.8 $\pm 7.8 \mathrm{ps}$ in the case of the ionic liquid, BMIM-PF6. Since the behavior of the bound sapphyrin is in marked contrast to what is seen for free sapphyrin, these transient absorption experiments lead us to conclude that the main driving force for the formation of the complex, namely $\pi$ stacking, is also operative in ionic liquids, as manifest in the red-shift in the spectral features and a shortening of the excited state lifetime. This supports the conclusion that the main driving force for the formation of the sapphyrin-nanotube complexes is enthalpic (i.e., driven by the presumed $\pi-\pi$ stacking interactions), rather than entropic, for which a greater media dependence would be expected.

The prediction that the nature of the association between sapphyrin and nanotubes would be a donor-acceptor interaction came from cyclic voltammetric analyses. Carried out in methylene chloride, these confirmed that the first oxidation potential of sapphyrin is $1.08 \mathrm{~V}$. Similarly, the reduction potential for the macrocycle was determined to be $-0.61 \mathrm{~V}$ (see Figure S2). On the other hand, the onset for oxidation of carbon nanotubes has been previously determined to be at ca. $0.6 \mathrm{~V} .{ }^{5}$ It is also known that there is a strong diameter dependence on the oxidation potential of SWNTs. ${ }^{13}$ Thus, these two entities are well positioned to act as donors and acceptors within a self-assembled noncovalent complex.

Considering the relevant potentials (i.e., sapphyrin reduction and SWNT oxidation), we approximate the energy of a charge separation process as $\sim 1.2 \mathrm{~V}$. The singlet excited state energy of sapphyrin, on the other hand, estimated as the average between longest wavelength absorption and shortest wavelength fluorescence, emerges as about $1.82 \mathrm{eV}$. Thus, the energy 
gap for electron transfer is close to $0.6 \mathrm{eV}$ at the oxidation onset value. Nonetheless, since the potential required for oxidation varies with SWNT diameter, the driving force for an electron transfer process involving the bound sapphyrin would be expected to vary somewhat with SWNT diameter and type.

One-electron reduction and one-electron oxidation experiments were carried out using radical species generated through pulse radiolysis. The resulting spectra are shown in Figure S3. The spectrum for the triplet excited state was obtained either photolytically or radiolytically (i.e., triplet energy transfer from the naphthalene triplet excited state), as shown in Figure S4.

Common to all species is ground state bleaching of the Soret- (i.e., $450 \mathrm{~nm}$ ) and Q-bands (i.e., 620 and $675 \mathrm{~nm}$ ). As far as the redox products are concerned, transient maxima around 425 / $840 \mathrm{~nm}$ and $480 \mathrm{~nm}$ were derived as important fingerprints of the radical anion and the radical cation, respectively. The triplet excited state, on the other hand, gives rise to maxima at 420 and $500 \mathrm{~nm}$.

With these assignments made, it was found that after the completion of the rapid singlet excited state decay, minima at 450 and $620 \mathrm{~nm}$ and maxima at 490 and $820 \mathrm{~nm}$ are observed. Thus, the product is a one-electron reduced rather than to a one-electron oxidized sapphyrin. This stands in marked contrast to what is normally seen in the case of the corresponding porphyrinbased systems, where electron transfer from the macrocycle to the SWNT is observed. ${ }^{14}$

Photoirradiation at $775 \mathrm{~nm}$ leads to the depopulation of electrons from the valence bands of the nanotubes and the build-up of a small broad positive absorption feature around $840 \mathrm{~nm}$ (see Figure S5). Coupled with the spectral information from the pulse radiolysis experiments, the femtosecond spectroscopic data thus provides strong support for the conclusion that upon irradiation, photo-induced electron transfer takes place from the SWNTs to the sapphyrin. This is shown graphically in Scheme 2.

\section{Conclusion}

Spectroscopic and microscopic investigations reveal that water-suspendable nanotubesapphyrin complexes are formed from the functionalized sapphyrin derivative 1. In particular, it was found that nanotubes serve to template the supramolecular association of sapphyrins on the surface of the nanotube under two very different sets of solvent conditions. The resulting modified nanotubes undergo photoexcited intramolecular electron transfer upon photoexcitation. Thus, the sapphyrin bound nanotubes act as antennae for light harvesting. Since donor-acceptor interactions play a key role in the development of photovoltaics, we propose that sapphyrin-nanotube hybrids, as well as those derived from other expanded porphyrins, could prove useful in the generation of nanotube-based electron transfer ensembles.

\section{Supplementary Material}

Refer to Web version on PubMed Central for supplementary material.

\section{ACKNOWLEDGMENTS}

The authors would like to thank the INEST group at PMUSA, the National Institutes of Health (grant GM 069492 to J.L.S.), and the Robert A. Welch Foundation (Grants E-680 and F-1018 to K.M.K. and J.L.S., respectively) for financial support of this project. 


\section{References}

1. a Kam NWS, Liu Z, Dai H. J. Am. Chem. Soc 2005;127:12492-12493. [PubMed: 16144388] b Sano M, Kamino A, Okamura J, Shinkai S. Nano Lett 2002;2:531-533. c Singh R, Pantarotto D, McCarthy D, Chaloin O, Hoebeke JP, Charalambos D, Briand J-P, Prato M, Bianco A, Kostarelos K. J. Am. Chem. Soc 2005;127:4388-96. [PubMed: 15783221]

2. Chichak KS, Star A, Altoe M, Virginia P, Stoddart JF. Small 2005;1:452-461. [PubMed: 17193471]

3. a Tagmatarchis N, Prato M, Guldi DM. Physica E 2005;29:546-550. b Guldi DM, Taieb H, Rahman GMA, Tagmatarchis N, Prato M. Adv. Mater 2005;17:871-875.

4. a Alvaro M, Atienzar P, De la Cruz P, Delgado JL, Troiani V, Garcia H, Langa F, Palkar A, Echegoyen L. J. Am. Chem. Soc 2006;128:6626-6635. [PubMed: 16704263] b Rahman GMA, Guldi DM,

Campidelli S, Prato M. J. Mater. Chem 2006;16:62-65. c Ehli C, Rahman GMA, Jux N, Domenico B, Guldi DM, Paolucci F, Marcaccio M, Paolucci D, Melle-Franco M, Zerbetto F, Campidelli S, Prato M. J. Am. Chem. Soc 2006;128:11222-11231. [PubMed: 16925441]

5. Sessler JL, Cyr MJ, Lynch V, McGhee E, Ibers JA. J. Am. Chem. Soc 1990;112:2810-13.

6. Sessler JL, Davis JM, Kral V, Kimbrough T, Lynch V. Org. Biomolec. Chem 2003;1:4113-4123.

7. Levanon H, Regev A, Michaeli S, Galili T, Cyr M, Sessler JL. Chem. Phys. Lett 1990;174:233-240.

8. Magda D, Crofts S, Sessler JL, Sansom P, Springs SL, Ohya Y. Tet. Lett 1997;38:5759-5762.

9. Sessler JL, Davis JM. Acc. Chem. Res 2001;34:989-997. [PubMed: 11747417]

10. Fukushima T, Kosaka A, Ishimura Y, Yamamoto T, Takigawa T, Ishii N, Aida T. Science 2003;300:2072-2074. [PubMed: 12829776]

11. Price BK, Hudson JL, Tour JM. J. Am. Chem. Soc 2005;127:14867-14870. [PubMed: 16231941]

12. Maiya BG, Cyr M, Harriman A, Sessler JL. J. Phys. Chem 1990;94:3597-3601.

13. a Zheng M, Diner B. J. Am. Chem. Soc 2004;126:15490-15494. [PubMed: 15563177] b Dukovic G, White BE, Zhou Z, Wang F, Jockusch S, Steigerwald ML, Heinz TF, Friesner RA, Turro NJ, Brus LE. J. Am. Chem. Soc 2004;126:15269-15276. [PubMed: 15548024]

14. Hecht DS, Ramirez RJA, Briman M, Artukovic E, Chichak KS, Stoddart JF, Grüner G. Nano Letters 2006;6:2031-2036. [PubMed: 16968021] 

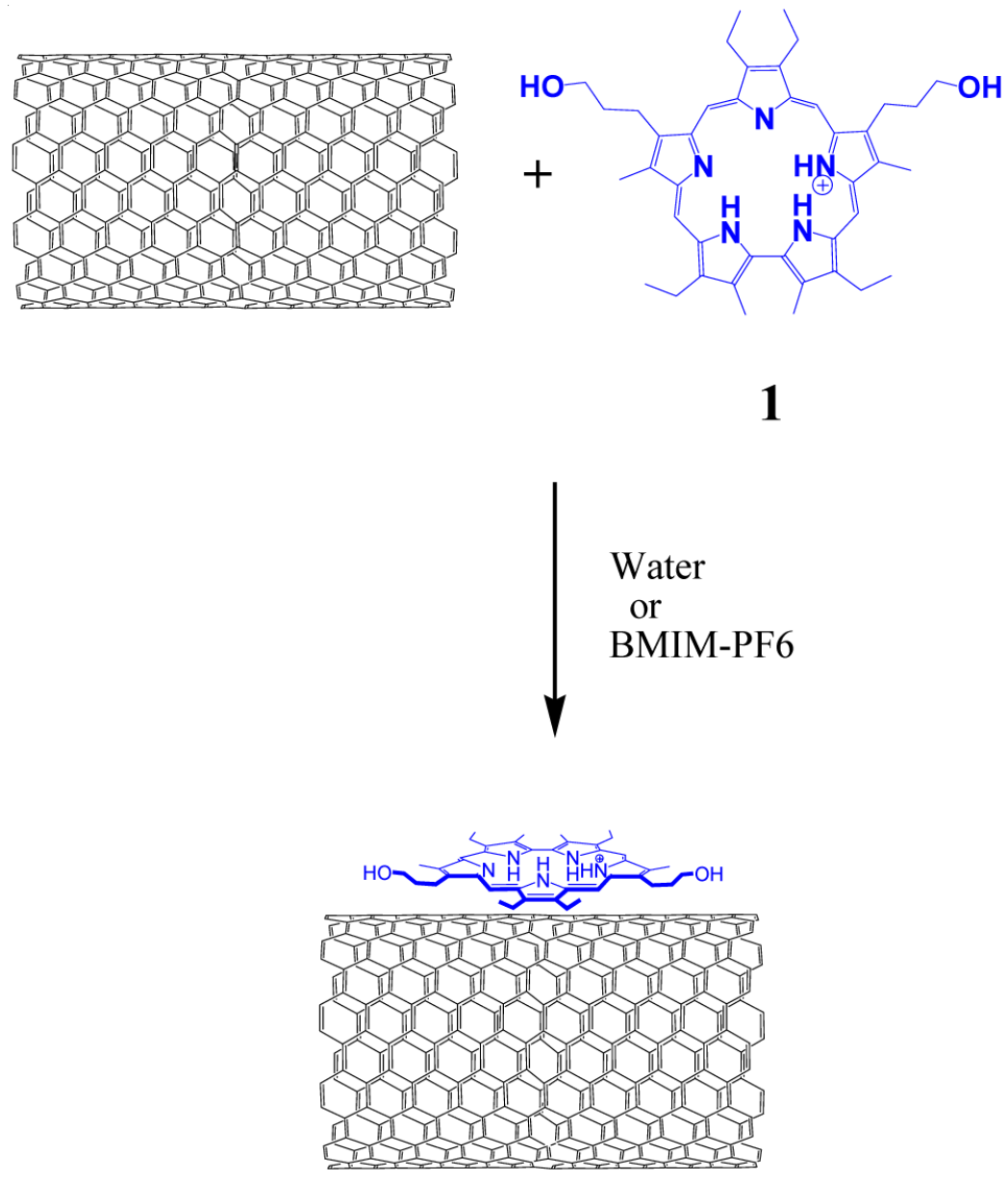

Scheme 1.

Schematic representation designed to illustrate the fact that supramolecular functionalization of SWNTs with sapphyrin takes place readily in both aqueous media and in an ionic liquid, 1butyl-3-methylimidazolium hexafluorophosphate (BMIM-PF6). In the ionic liquid, supramolecular functionalization was achieved without sonication. 


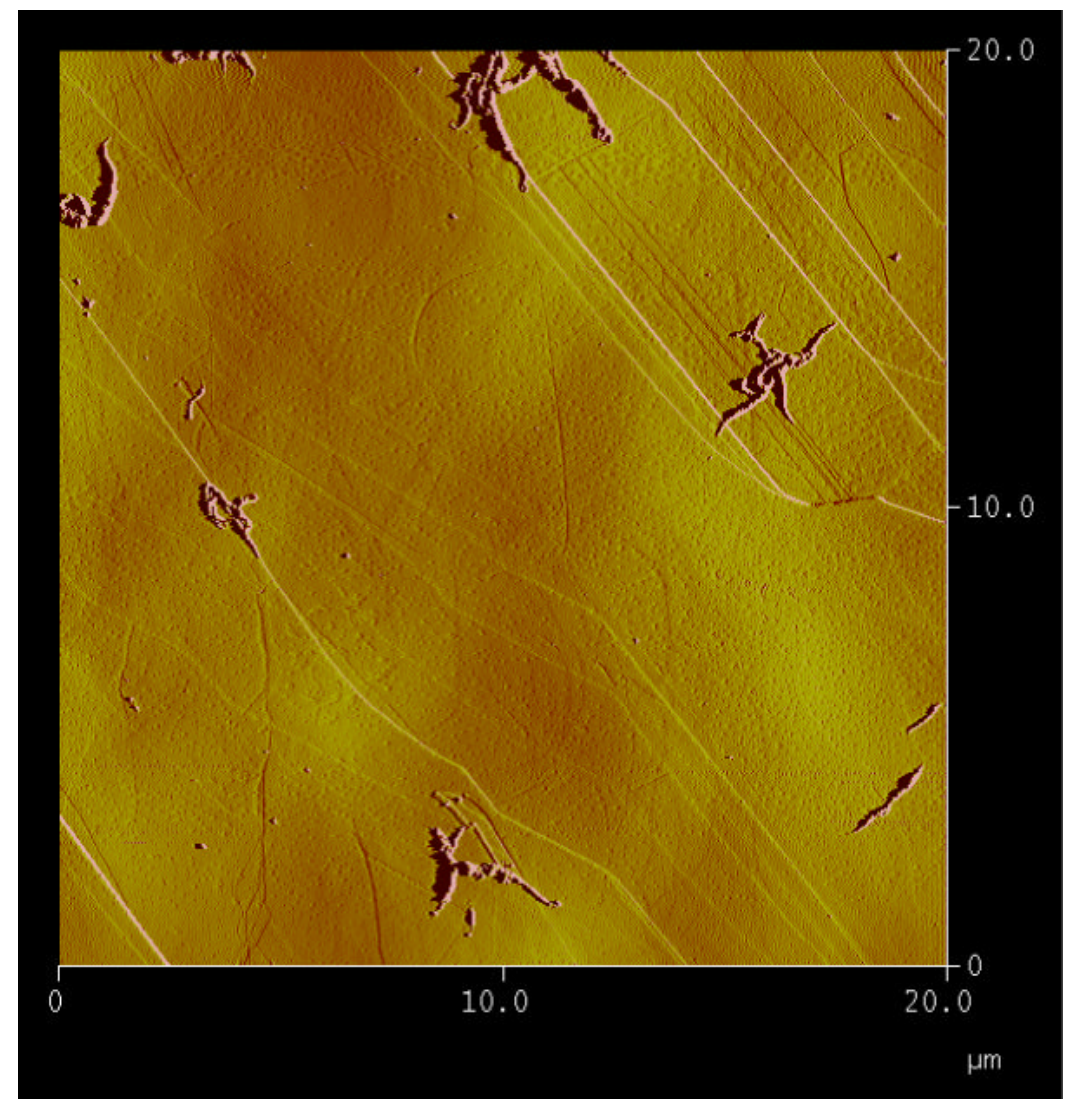

Figure 1.

An atomic force microscopic image (tapping mode) of sapphyrin nanotubes spin-coated onto HOPG. Mostly bundles are observed on the substrate, along with some individual nanotubes. 


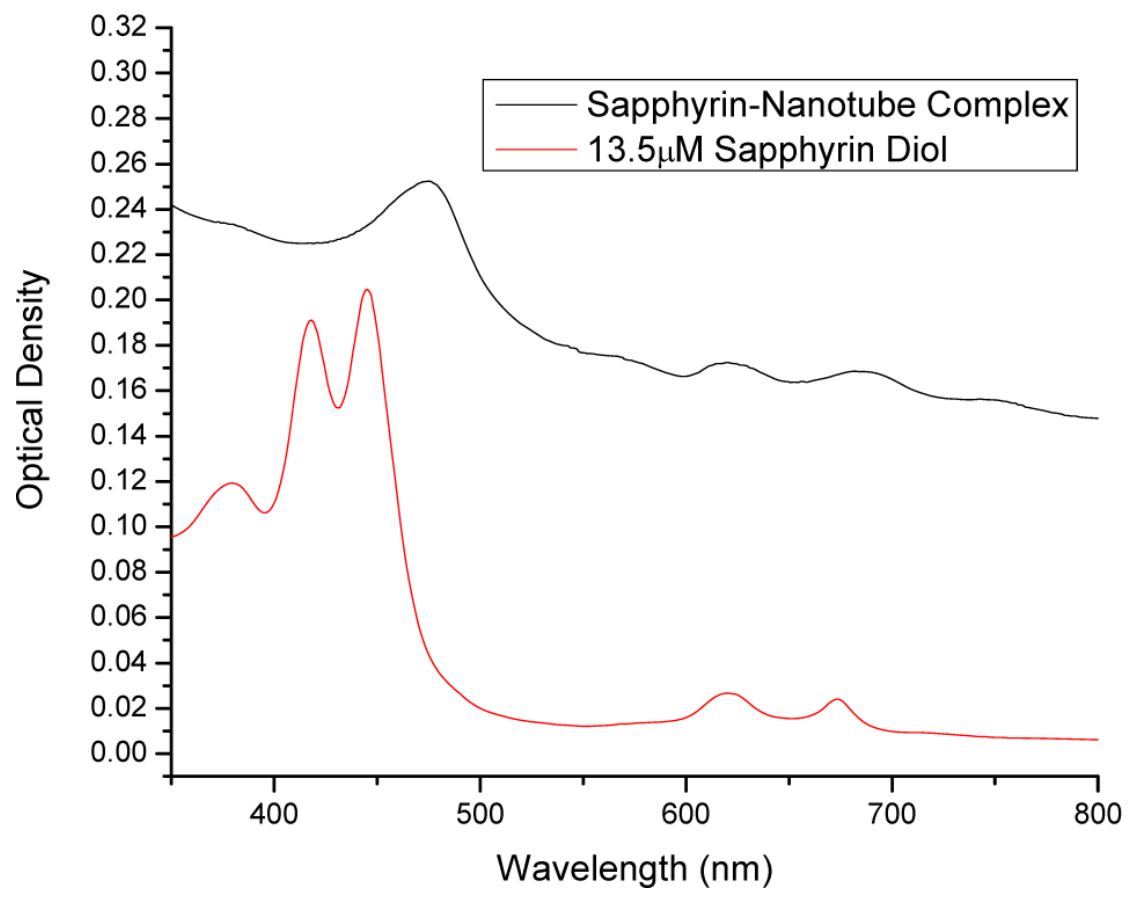

Figure 2.

Absorption spectra of sapphyrin 1 and aqueous suspensions of sapphyrin-functionalized nanotubes. Monomeric sapphyrin displays a Soret-like maximum at $450 \mathrm{~nm}$ that is red-shifted to $477 \mathrm{~nm}$ when sapphyrin is allowed to interact with a nanotube, as discussed in the text. 


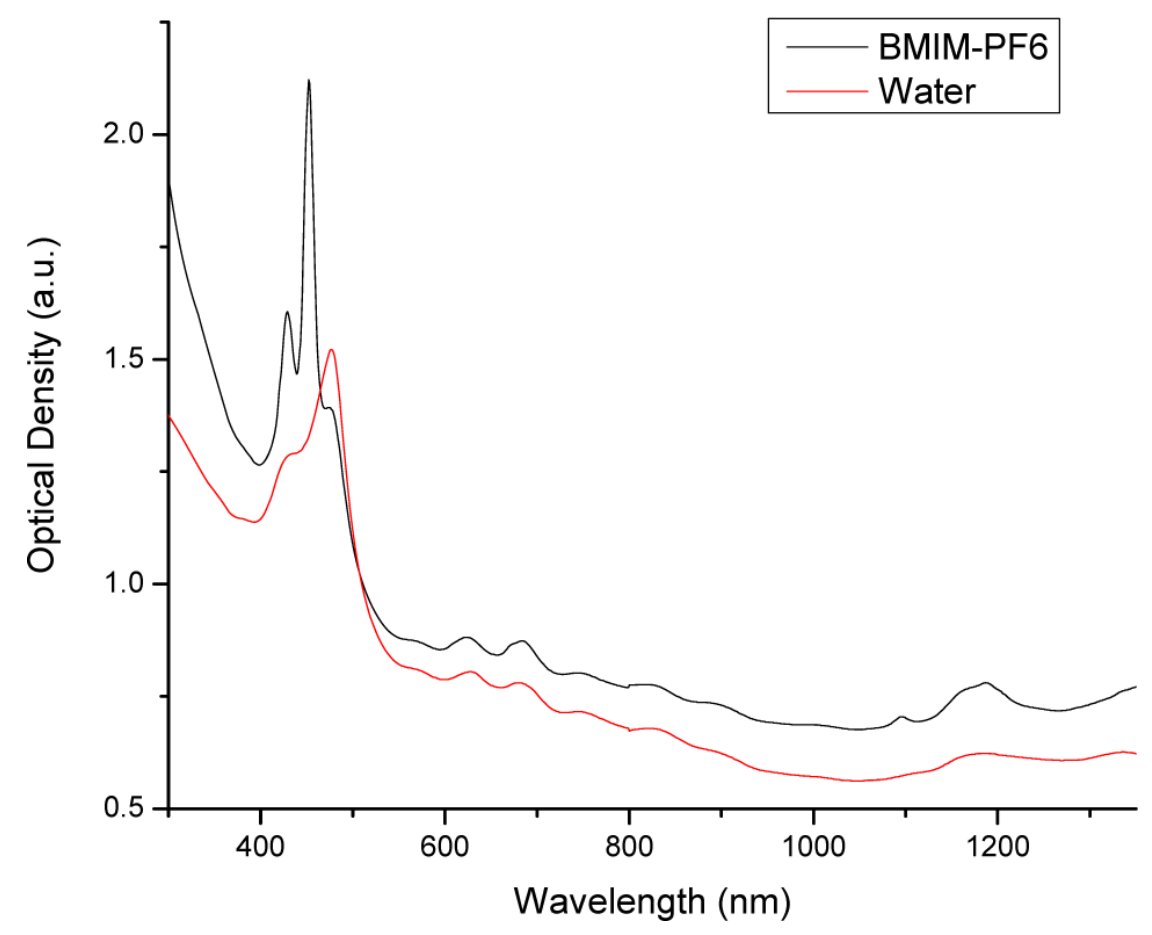

Figure 3.

Steady-state absorption spectra of sapphyrin-nanotube nanohybrids acquired in water and in the ionic liquid BMIM-PF6. A shoulder in the spectral region around $470 \mathrm{~nm}$ is observed in the case of mixtures of sapphyrin and SWNTs recorded in BMIM-PF6. Transient absorption measurements reveal that photoexcitation of the sample in BMIM-PF6 leads to bleaching at $481 \mathrm{~nm}$, an effect that is rationalized in terms of changes in the Soret-like band of the sapphyrin bound to the nanotube. 


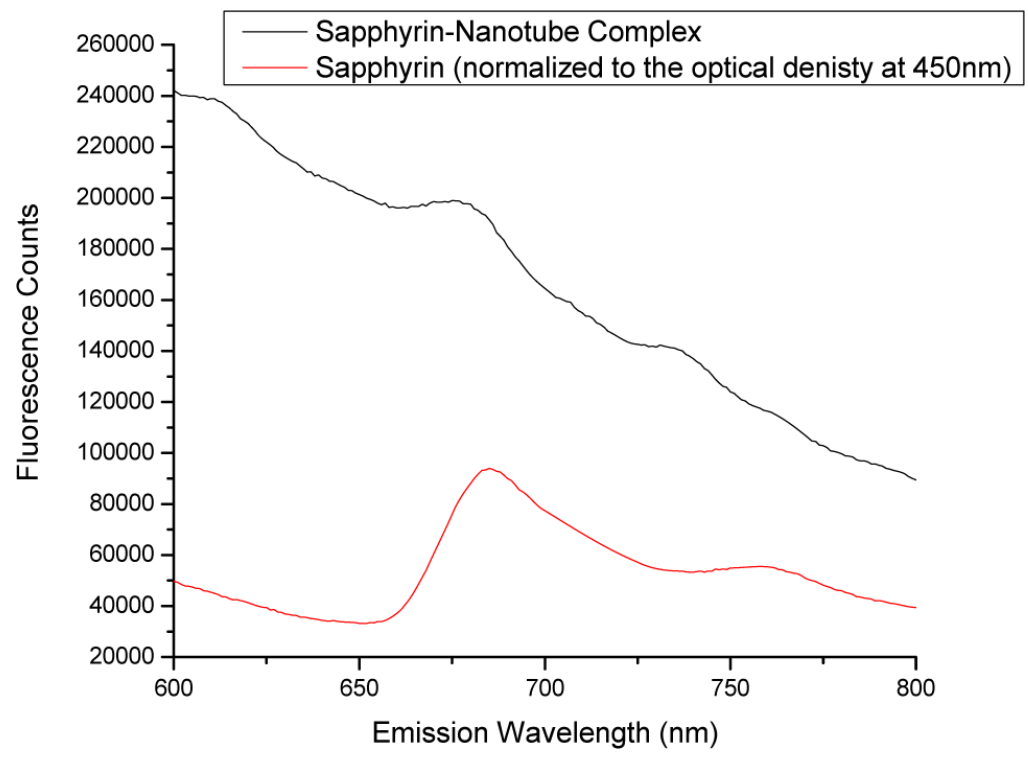

Figure 4.

Fluorescence emission spectra recorded after excitation at $450 \mathrm{~nm}$. Both samples were adjusted to have the same absorption at $450 \mathrm{~nm}$. The major emission peak at $687 \mathrm{~nm}$ is considerably diminished and blue shifted in the nanotube sample, while an additional peak at $740 \mathrm{~nm}$ is observed. This additional peak is ascribed to nanotube fluorescence. This latter emission explains why the fluorescence background is higher for the sappphyrin nanotube samples than for sapphyrin alone. 


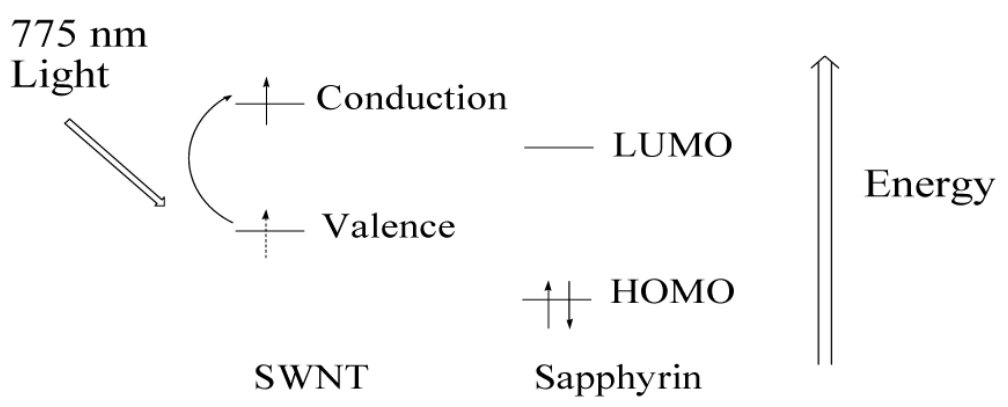

(a) Irradiation at $775 \mathrm{~nm}$

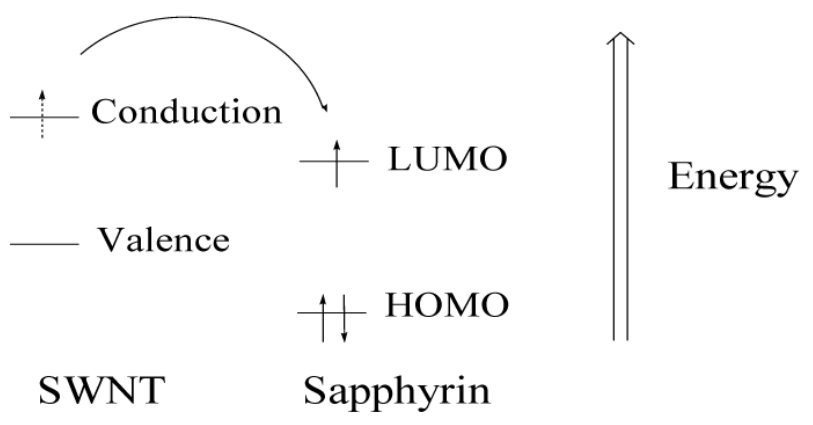

(b) Electron Transfer to Sapphyrin from SWNTs

Scheme 2.

Energetics of the observed photoinduced electron transfer. Irradiation at $775 \mathrm{~nm}$ results in hole formation on SWNTs and electron donation to sapphyrin. 